\title{
From the New Editor-in-Chief
}

\author{
Jonathan N. Sachs ${ }^{1}$
}

Published online: 26 May 2016

(C) Springer Science+Business Media New York 2016

I am pleased to introduce myself as the new Editor-in-Chief of Applied Biochemistry and Biotechnology $(A B A B)$. While new to $A B A B$, I have served as Associate Editor and then Managing Editor for another Springer journal, The Journal of Membrane Biology. These are exciting but critical times in the development of biotechnology as an academic field, and we aim to place our journal at the center of this growth over the coming decade. Biotechnology is here to stay as a major economic driver throughout the modern world, and significant challenges will be met through increasingly inter-woven collaboration between academic science and industry. In particular, this journal will strive to keep pace with this and to serve the community by publishing cutting edge articles in areas including, among others: energy and environmental sustainability; food production and safety; and innovative biotechnologies in heath and medicine for an increasingly globalized and economically stratified world. One main goal is to raise the journal's profile and impact factor through a multi-faceted process of rigorous peer review, anti-plagiarism vigilance, and a transformation in the quality of research articles submitted to and published by the journal.

You may have already noticed some changes in the journal. In particular, the journal is now divided into five topical sections, each of which is led by one of our dedicated Associate Editors:

Biofuels and biochemicals from renewable bioresources (Associate Editor: Jie Bao)

Renewable feedstocks, especially the non-food biomass resources. The section includes novel microbes, enzymes, processes involved in the production of biofuels, and biochemicals from renewable bioresources with the focus on non-food biomass resources.

\section{Biological sensing and bioremediation (Associate Editor: Yu Lei)}

All aspects of biological sensing and bioremediation. Development and application of any novel detection and remediation technologies/processes employing biological elements (e.g., tissue, microorganisms, organelles, cell receptors, enzymes, antibodies, nucleic acids, etc.) or their biomimetic analogues.

Jonathan N. Sachs

sachsabab@gmail.com

1 Department of Biomedical Engineering, University of Minnesota, Minneapolis, MN 55455, USA 


\section{Applied biotechnology for medical diagnosis and therapeutics (Associate Editor: Chenzhong Li) \\ Biomedical sciences and biomedical engineering with specific interests in biomedical devices and sensors, nanomedicine, biomaterials, regenerative medicine, bioelectronics, bio-imaging, and instrumentations for medical diagnosis and therapeutics.}

\section{Biocatalysis for organic synthesis (Associate Editor: Ye Ni)}

Novel and significant advances in biocatalysis for organic synthesis, including chemo-, regio-, and enantioselective biotransformation, screening and engineering of novel enzymes for biocatalysis, biocatalyst immobilization, and nonaqueous biocatalysis.

\section{Environmental genomics (Associate Editor: Hemant Purohit)}

Environmental bio-processes, waste water treatment, plant microbe interactions, genome sequence data and prediction of pathways, designing and development of genomics tools and its application, and gene expression analysis.

These sections reflect the current scope of the journal but may change or increase as we expand or modify the scope in the coming years. We are also aggressively increasing the impact expectation for submitted and published articles, a process in which we will consistently engage the Editorial Board.

I and the Associate Editors are interested in your feedback and questions, so please pass any along to me. We look forward to your continued engagement with the journal.

Jonathan N. Sachs

Editor-in-Chief

Applied Biochemistry \& Biotechnology

sachsabab@gmail.com 